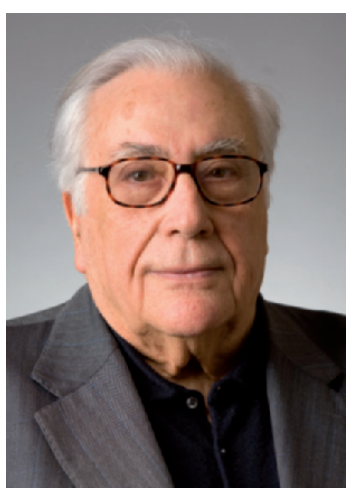

_M.M. Samama

Groupe Hospitalier Broca-Cochin, Hôtel-Dieu, Paris (France) Biomnis, Ivry-sur-Seine (France) E-mail : meyermichel.samama@biomnis.com

\section{Évolution et innovations en hémostase et anticoagulation}

Depuis plus de cinquante ans, les antagonistes de la vitamine K (AVK) constituent la seule famille de médicaments anticoagulants actifs par voie orale. Ils agissent en inhibant la synthèse de plusieurs facteurs de la coagulation. Deux nouveaux anticoagulants actifs par voie orale ont apporté une innovation thérapeutique importante. Ils inhibent spécifiquement un seul facteur de la coagulation. II s'agit du Dabigatran étexilate, inhibiteur direct du Facteur Ila ou thrombine, et du Rivaroxaban, inhibiteur direct du Facteur Xa. D'autres molécules sont en cours de développement (Apixaban, Edoxaban, etc...). Les traitements avec les nouveaux anticoagulants dispensent le patient d'une surveillance régulière de la coagulation. II existe néanmoins des circonstances cliniques où une mesure de l'anticoagulation induite par ces nouveaux médicaments devient utile (accident hémorragique, échec du traitement, chirurgie urgente, mauvaise adhérence au traitement...). Bien plus, si des examens de la coagulation sont faits dans le cadre d'autres problèmes cliniques, les résultats doivent pouvoir être interprétés valablement par le biologiste. En effet, ces anticoagulants peuvent modifier les résultats de nombreux examens de coagulation. Les deux premiers articles de ce numéro sont consacrés à une mise au point sur les caractéristiques pharmacologiques et le retentissement sur les examens de la coagulation de ces nouvelles molécules. L'étude de l'activité anti-Xa longtemps réservée aux héparines trouve un intérêt dans l'étude des anticoagulants inhibiteurs directs du Facteur Xa : un article est consacré à cette mesure. Les héparines de bas poids moléculaire ou HBPMs sont utilisées avec succès depuis plus d'un quart de siècle. Elles sont essentiellement éliminées par le rein et leur utilisation doit être ajustée ou récusée en cas d'insuffisance rénale. Un chapitre discute cette éventualité. Enfin, parmi les dosages des facteurs de la coagulation, celui du Facteur VIII, longtemps restreint au diagnostic de l'hémophilie, connaît aujourd'hui de nombreuses applications qui sont développées dans ce chapitre. II nous a paru enfin souhaitable dans ce dossier d'actualités en hémostase et anticoagulation d'analyser les progrès des examens de laboratoire, leur évolution, leur limites et les innovations permettant d'espérer une meilleure analyse des altérations de la coagulation et en particulier de la recherche d'une hypercoagulation. • 